\title{
Saccharification of lignocellulosic biomass under mild condition using ionic liquid
}

\section{AUTHOR(S):}

Muranaka, Yosuke; Suzuki, Tatsuya; Hasegawa, Isao; Mae, Kazuhiro

\section{CITATION:}

Muranaka, Yosuke ...[et al]. Saccharification of lignocellulosic biomass under mild condition using ionic liquid. Journal of Chemical Engineering of Japan 2015, 48(9): 774781

\section{ISSUE DATE:}

2015-09

URL:

http://hdl.handle.net/2433/217398

\section{RIGHT:}

(c) 2015 The Society of Chemical Engineers, Japan.; Publisher permitted posting the accepted manuscript on this repository. 化学工学会の許可 を得て登録しています。 


\title{
Saccharification of Lignocellulosic Biomass under Mild Condition Using Ionic Liquid
}

\author{
Yosuke MuranaKa, Tatsuya SuzuKI, Isao HaSEgawa and Kazuhiro \\ $\mathrm{MAE}^{*}$ \\ Department of Chemical Engineering, Kyoto University, Kyoto-daigaku \\ Katsura, Nishikyo-ku, Kyoto 615-8510, Japan
}

Key words: Pretreatment, Biomass, Ionic liquid, Saccharification

Biomass is expected to be an alternative resources to fossil resources. In this study, the development of biomass conversion method into the valuable chemical, reducing sugar, was examined. For the conversion, thermochemical technology was focused on for its advantage of short reaction time, and ionic liquid was focused as reagent to overcome an unpreferable disadvantage of thermochemical technology, which is, the low selectivity. Cedar and crystalline cellulose were pretreated with ionic liquid for a reforming into desirable precursors of reducing sugar. Especially when they were pretreated by 1-Ethyl-3-methylimidazolium methylphosphonate at $150{ }^{\circ} \mathrm{C}$ for $1 \mathrm{~h}$, pretreatment worked effectively by decreasing the crystallinity of samples. Pretreated cedar and crystalline cellulose were converted into reducing sugar under the hydrothermal condition, respectively, by 39 C-\% and 90 C-\%. Recovery of ionic liquid was also examined. When cedar was used as a material, lignin was dissolved into ionic liquid through pretreatment, which was undesirable because of their difficult separation. When crystalline cellulose was used as a material, $98.3 \%$ of ionic liquid was recovered after the conversion with the highest yield of reducing sugar (90 C-\%).

\section{Introduction}

Biomass has a potential to be an alternative resource to crude oil. However, it has the problems of high collecting cost and limited available amount. Therefore, to make biorefinery feasible, its superiority to oil refinery needs to be considered. Crude oil usage is mainly categorized into energy use and production of chemicals. For the production of chemicals, crude oil is usually converted into ethylene or propylene once, and then converted into chemicals. These chemicals include oxygen containing chemicals such as ethylene oxide or acetaldehyde. To produce these oxygen containing chemicals from ethylene or propylene, an oxidation process is required. However, an oxidation process generally has the problem of very low selectivity. On

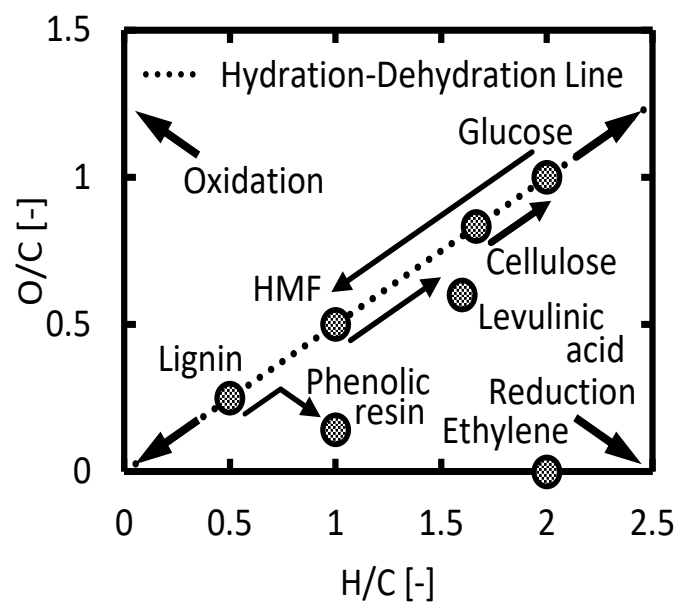

Fig. 1 Conversion pathways of biomass components and ethylene into chemicals the other hand, biomass already contains oxygen in its structure. Although the oxygen is considered as disadvantage in the point of energy use because it decreases the heat of combustion, it could be considered as huge superiority of biomass for the production of chemicals. Figure 1 shows the values of $\mathrm{O} / \mathrm{C}$ plotted against the $\mathrm{H} / \mathrm{C}$ based on the elemental composition of chemicals. Contrary to the crude oil derivatives, biomass derivatives require dehydration, hydration or reduction which possibly enable the selectivity to be $100 \%$. In addition, biomass-derived products can be used as energy resources in the end of their use through combustion. Thus, the use of biomass as the material for the production of chemicals seems to be more reasonable than the direct use as the energy resource. Biomass mainly consists of three components; cellulose, hemicellulose and lignin. About woody biomass, the main component is cellulose, which is the most abundant natural resource on this planet. This means the development of the new conversion method of cellulose is essential for the conversion of biomass into valuable chemicals. In this study, the effective conversion method of biomass or cellulose into reducing sugar, the product obtainable by the hydrolysis of cellulose, was investigated. Biomass is normally converted into reducing sugar by thermochemical technology or biochemical technology. Biochemical technology has huge advantage of high selectivity. This is the indispensable advantage for the utilization of biomass as an alternative resource. However, biochemical 
technology also has the disadvantages of long reaction time and high cost due to the expensive enzymes, the need of sewage treatment and complicated control of reactor. Consequently, the development of thermochemical technology for the effective conversion of biomass was investigated. The advantage of thermochemical technology is that it does not require long reaction time. On the other hand, there are some disadvantages as well such as low selectivity and huge energy consumption. To overcome these problems, the unique character of ionic liquid was focused on. Ionic liquid is the salt which is able to exist as liquid under relatively low temperature (Forsyth et al., 2004). Although the melting point of salts is generally high, it is possible to be lowered by making the radius of cation and anion bigger and weakening the electrostatic force between them. Despite the weakened electrostatic force between ions, the force is still strong enough to have features such as non-volatility, incombustibility and ionic conductivity. For its unique character, ionic liquid is used for many applications such as lubricant, solvent or electrolyte, and there have been many studies on ionic liquid recently, e.g., Phillips et al. (2004), Liu et al. (2012) and Barthel et al. (2006). Swatloski et al. (2002) and Sievers et al. (2009) reported that, among the many types of ionic liquid, the types containing the imidazole group are effective on solubilization of cellulose and biomass breaking the rigid structure of cellulose. For example, 1-Ethyl-3-methylimidazolium bromide ([EMIM]Br) breaks the rigid structure of cellulose and solubilizes biomass. Cellulose solubilized by this ionic liquid is known to be recovered by adding another liquid which has no ability of dissolving cellulose (Vo et al., 2012). Because of this unique characteristic, cellulose is supposed to be easily uncrystallized and separated from ionic liquid. Another example is 1-Ethyl-3-methylimidazolium methylphosphonate ([EMIM]P). [EMIM]P has high ability of breaking the rigid structure of cellulose, and it changes cellulose to water-soluble cellulose. Uju et al. (2012) reported that solubilization of cellulose enables some reagents such as enzymes or acids to attack the active cites of cellulose more effectively. For example, Dadi et al. (2006) reported that cellulose could be converted into reducing sugar in the yield of $90 \%$ by using ionic liquid. However, in this efficient method, cellulose is converted by biochemical technology and it requires reaction time of $50 \mathrm{~h}$. Another example using ionic liquid, via thermochemical technology, was reported by $\mathrm{Li}$ et al. (2007), which enables cellulose conversion into reducing sugar reach $77 \%$ by the treatment of $9 \mathrm{~h}$ at $100{ }^{\circ} \mathrm{C}$. However, this method introduces acidic catalyst in the process of hydrolysis, which requires the additional separation process. In this study, thus, we pretreated biomass or cellulose with ionic liquid at first to solubilize them and to make desirable precursors highly selective for reducing sugar, and then degraded obtained precursors to the reducing sugar under the hydrothermal condition without using any reagent except for ionic liquid and water.

\section{Experimental}

All the experimental procedures explained in this section are briefly summarized in Figure 2.

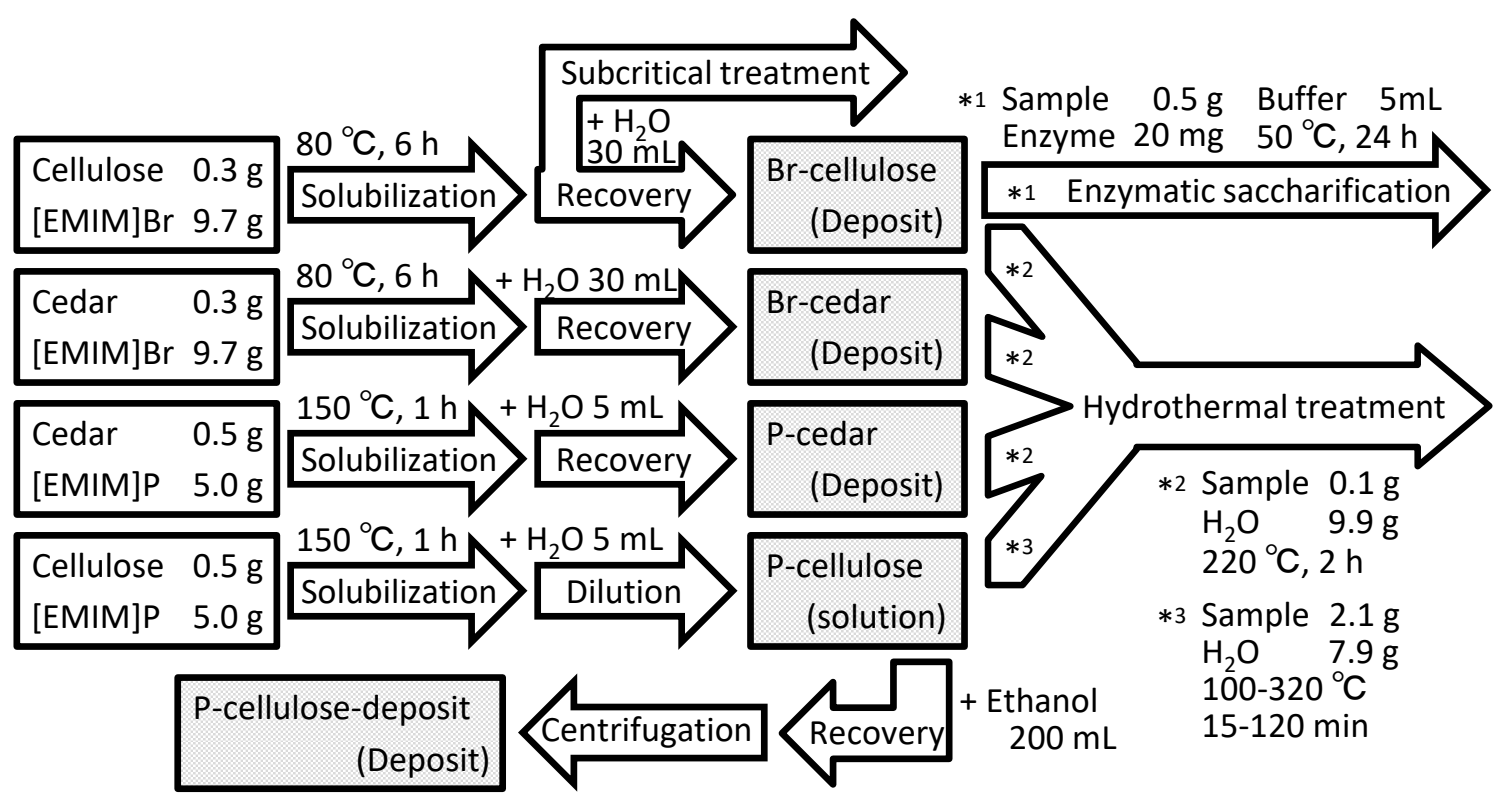

Fig. 2 Reaction flowchart (*1 and ${ }^{* 2}$ were conducted using crystalline cellulose or amorphous cellulose as well) 
Table 1 Ultimate analyses of samples used

\begin{tabular}{lcccc}
\hline & $\mathrm{C}$ & $\mathrm{H}$ & $\mathrm{O}$ (diff.) & $\mathrm{H} / \mathrm{C}$ \\
{$[\mathrm{wt} \%]$} & {$[\mathrm{wt} \%]$} & {$[\mathrm{wt} \%]$} & {$[-]$} \\
\hline Crystalline Cellulose & 42.95 & 6.20 & 50.85 & 0.14 \\
Amorphous Cellulose & 43.37 & 6.12 & 50.52 & 0.14 \\
Cedar & 46.87 & 4.84 & 48.28 & 0.10 \\
\hline
\end{tabular}

\subsection{Materials}

For the efficient saccharification of cellulose, biomass and crystalline cellulose were pretreated with ionic liquid. [EMIM]Br or [EMIM]P was used as ionic liquid and cedar (cryptomeria japonica) was used as the biomass sample. [EMIM]Br was purchased from Wako Pure Chemical Industries, Ltd. (Tokyo, Japan) and [EMIM]P was purchased from KANTO CHEMICAL CO, INC (Tokyo, Janapn). Cedar was purchased from Kobo Mokuyo-Daiku (Tokyo, Japan) and pulverized using crusher (AS ONE Corporation) to particles of under $500 \mu \mathrm{m}$. Crystalline cellulose and amorphous cellulose were obtained from Kao Corporation (Tokyo, Japan). The ultimate analyses of pulverized cedar, crystalline cellulose and amorphous cellulose are listed in Table 1.

\subsection{Pretreatment}

The pretreatments were performed by using glass bottle with an internal volume of $50 \mathrm{~cm}^{3}$. When [EMIM]Br was used as the ionic liquid, $0.3 \mathrm{~g}$ of cedar or crystalline cellulose and $9.7 \mathrm{~g}$ of [EMIM]Br were mixed in a glass bottle and put in a water bath preheated to $80{ }^{\circ} \mathrm{C}$ for $6 \mathrm{~h}$ under stirring. Immediately after heating, the products were cooled, $30 \mathrm{~mL}$ of pure water was added for the deposition of the solute, and the mixture was filtered by suction. The deposit was rinsed with pure water, dried in vacuo, and then analyzed by CHNS elemental analysis, thermogravimetric(TG) analysis and X-ray diffraction(XRD). The deposit is denoted as Br-cellulose or Br-cedar hereafter in this article. When [EMIM]P was used as the ionic liquid, $0.5 \mathrm{~g}$ of cedar or crystalline cellulose and $5.0 \mathrm{~g}$ of [EMIM]P were mixed in a glass bottle and put in an oil bath preheated to $150{ }^{\circ} \mathrm{C}$ for $1 \mathrm{~h}$ under stirring. Immediately after heating, the products were cooled and $5 \mathrm{~mL}$ of pure water was added. For cedar sample, the products were filtered by suction and rinsed with pure water. The residue is donated as P-cedar hereafter in this article. For the cellulose sample, after adding $5 \mathrm{~mL}$ of water, $200 \mathrm{~mL}$ of ethanol was added for the recovery because crystalline cellulose was converted into water-soluble components by [EMIM]P, and then the deposit was separated by centrifuge. The deposit and the solubilized crystalline cellulose without the addition of ethanol are denoted as P-cellulose-deposit and P-cellulose hereafter in this article. The solid samples, P-cedar and
P-cellulose-deposit, were dried in vacuo, and then analyzed by CHNS elemental analysis, TG analysis and XRD.

\subsection{Enzymatic Saccharification}

To investigate whether the pretreatment with ionic liquid works effectively, enzymatic sacchrification was conducted on Br-cellulose, crystalline cellulose and amorphous cellulose. Aspergillus was chosen for enzyme. $0.5 \mathrm{~g}$ of cellulose sample (Br-cellulose, crystalline cellulose or amorphous cellulose), $20 \mathrm{mg}$ of enzyme and $5 \mathrm{~mL}$ of citrate buffer solution $(0.1 \mathrm{~mol} / \mathrm{L}, \mathrm{pH}=5.0)$ were mixed in $30 \mathrm{~cm}^{3}$ glass bottle and heated at $50{ }^{\circ} \mathrm{C}$ for $24 \mathrm{~h}$ in a water bath under stirring. The products were filtered by suction after the reaction and filtrates were diluted with pure water for the analysis by high performance liquid chromatography (HPLC).

\subsection{Recovery of Solubilized Crystalline Cellulose by [EMIM]Br with Subcritical Water}

The direct degradation of solubilized crystalline cellulose by [EMIM]Br was examined using subcritical water for the recovery instead. Solubilized cellulose by [EMIM]Br was prepared as the section 1.2. Instead of adding $30 \mathrm{~mL}$ of water, 30 $\mathrm{mL}$ of dimethyl sulfoxide (DMSO) was added to lower the viscosity of the solution for the flow reaction process. The sample was collided with subcritical water at $250{ }^{\circ} \mathrm{C}$ under the pressure of $20 \mathrm{MPa}$. The flow rate of the supplied sample or pure water was $1.5 \mathrm{~mL} / \mathrm{min}$ or $0.5 \mathrm{~mL} / \mathrm{min}$, respectively. The products contained dispersed particles, which were separated by centrifuge. The supernatant liquid was diluted with pure water by $100 \mathrm{~mL}$ and analyzed by HPLC.

\subsection{Hydrothermal Degradation}

The hydrothermal treatments were performed by using Swagelok (316 stainless steel) batch reactor with an internal volume of $30 \mathrm{~cm}^{3}$ or $10 \mathrm{~cm}^{3} .0 .1 \mathrm{~g}$ of Br-cedar, P-cedar or Br-cellulose and $9.9 \mathrm{~g}$ of pure water were mixed in a sealed batch reactor and put in an oil bath preheated to $220{ }^{\circ} \mathrm{C}$ for $2 \mathrm{~h}$. The reactor was cooled in a water bath after the reaction and then the products were filtered by suction. Filtrates were diluted with pure water by $100 \mathrm{~mL}$ and analyzed by 3,5-dinitrosalicylic acid (DNS) method. For comparison, experiments with raw materials (cedar, crystalline cellulose or amorphous cellulose) were conducted according to the same method. Because the recovery of P-cellulose-deposit requires plenty amounts of ethanol, the hydrothermal treatment on crystalline cellulose pretreated with [EMIM]P was conducted using solubilized sample. $2.1 \mathrm{~g}$ of P-cellulose (which consist of $1.0 \mathrm{~g}$ of [EMIM]P, $1.0 \mathrm{~g}$ of pure water and $0.10 \mathrm{~g}$ of cellulose) and $7.9 \mathrm{~g}$ of water were mixed in a sealed batch reactor and put in an oil bath preheated to $100-320{ }^{\circ} \mathrm{C}$ for $15-120$ 
min. The cooling process, filtration process and analysis were conducted according to the same method as the other samples.

\subsection{Analyses of Products}

Ultimate analysis of the samples was performed using a CHNS elemental analyzer (BEL Japan, Inc., ECS4010). The analysis of pyrolysis profile of solid sample was performed using a TG analyzer (Shimadzu, TGA-50). Crystallinity of samples was measured by XRD (Rigaku Corporation). For the

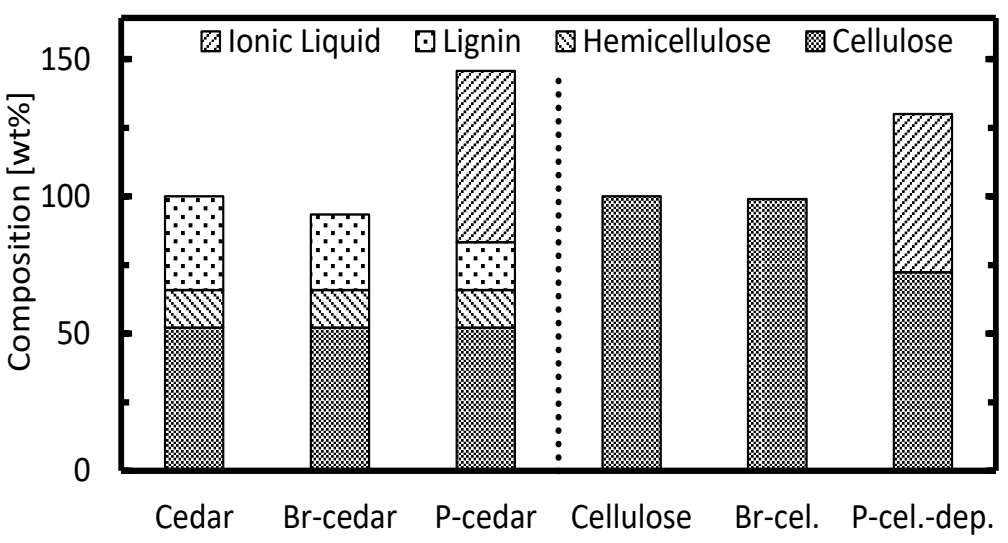

Fig. 3 Compositions of raw materials and pretreated samples organic acid analysis, an aqueous solution containing $951 \mathrm{mg} / \mathrm{L}$ of $p$-toluene sulfonic acid, $4185 \mathrm{mg} / \mathrm{L}$ of Bis-Tris, and $29 \mathrm{mg} / \mathrm{L}$ of ethylenediamietetraacetic acid was used as the eluent, and it was fed at $0.8 \mathrm{~mL} / \mathrm{min}$ to the HPLC equipped with a sulfonated polystyrene gel column (Shim-pack SCR-102H) and an electric conductivity detector (Shimadzu, CDD-6A). The yield of each component of organic acids estimated from the above measurements was represented on the basis of dry and ash-free samples. The yield of reducing sugar was calculated through the analysis by DNS method. DNS reagent was prepared by dissolving $4 \mathrm{~g}$ of $\mathrm{NaOH}, 1.25 \mathrm{~g}$ of dinitrosalicylic acid and $75 \mathrm{~g}$ of poptassium sodium tartrate into $400 \mathrm{~mL}$ of pure water. The mixture of the reagent and sample, $2 \mathrm{~mL}$ of each, was heated with boiling water for $10 \mathrm{~min}$, cooled with iced water for $2 \mathrm{~min}$, and then $3 \mathrm{~mL}$ of pure water was added. Absorbance for $540 \mathrm{~nm}$ of ultraviolet was measured using UV-Visible

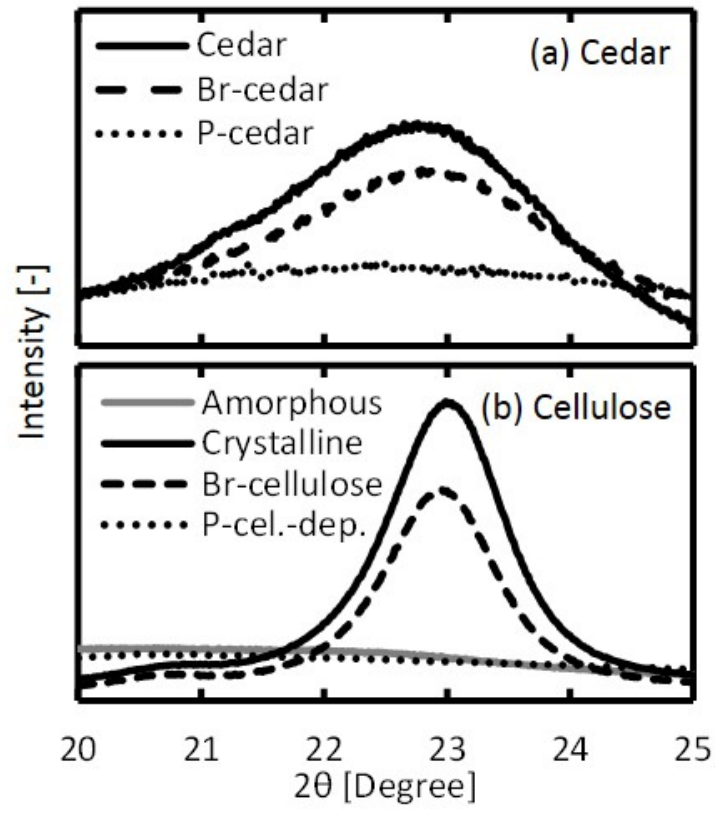

Fig. 4 XRD patterns of samples; (a) Cedar, (b) Cellulose

spectrophotometer (Shimadzu, UVmini-1240) for the yield calculation. The yield of reducing sugar was calculated by HPLC as well. An solution consists of $70 \mathrm{wt} \%$ of acetonitrile and $30 \mathrm{wt} \%$ of pure water was used as the eluent, and it was fed at $0.7 \mathrm{~mL} / \mathrm{min}$ to the HPLC equipped with a sulfonated styrene divinyl benzene copolymer column (Shodex, RSpak DC-613) and a charged aerosol detector (Dionex, Corona).

\section{Results and Discussion}

\subsection{Pretreatment of Cedar}

After the pretreatment of cedar with [EMIM]Br, $93.4 \mathrm{wt} \%$ of cedar was recovered as the deposit (Br-cedar). The compositions of the residue is shown in Figure 3. There was some weight loss through the pretreatment. This loss was considered as lignin-derived component because the hydrothermal degradation on filtrates was conducted and no cellulose-derived or hemicellulose-derived

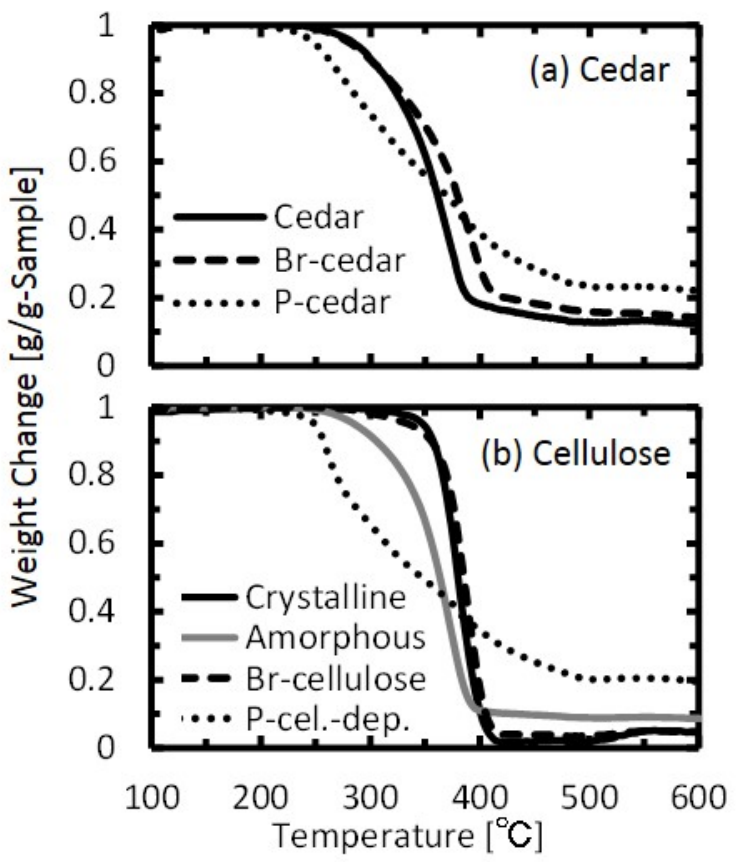

Fig. 5 TG curves of samples; (a) Cedar, (b) Cellulose 
Table 2 Elemental compositions of samples

\begin{tabular}{lccccc}
\hline & $\mathrm{C}$ & $\mathrm{H}$ & $\begin{array}{c}\text { O (diff.) } \\
\text { [wt\%] }\end{array}$ & $\mathrm{N}$ & $\mathrm{P}$ \\
\hline Br-cellulose & 44.10 & 6.10 & 49.80 & - & - \\
P-cellulose-deposit & 38.16 & 7.13 & 42.83 & 5.64 & 6.24 \\
Br-cedar & 49.82 & 4.34 & 45.79 & 0.05 & - \\
P-cedar & 40.80 & 6.02 & 42.25 & 5.19 & 5.74 \\
\hline
\end{tabular}

component was produced. Through the pretreatment with [EMIM]P, cedar was converted into the slurry-like components. The dry residue, P-cedar, was recovered by $145.8 \mathrm{wt} \%$ on the basis of the amount of cedar used. According to the analysis by CHNS elemental analysis and TG analysis, it was clarified that 83.3 wt $\%$ out of $145.8 \mathrm{wt} \%$ derived from cedar and rest of $62.5 \mathrm{wt} \%$ derived from [EMIM]P. To clarify the component of solubilized $16.7 \mathrm{wt} \%$, the filtrates were treated under hydrothermal condition. Through the reaction, no cellulose-derived or hemicellulose-derived component was produced. According to this fact, the conversion into P-cedar was calculated as there were no decreases of cellulose and hemicellulose, and only lignin was lost as soluble component by the pretreatment. Figure 4 (a) and Figure 5 (a) show the XRD patterns, and the TG curves of the samples and the products. Table 2 shows the elemental compositions of Br-cedar and $\mathrm{P}$-cedar, where the values $\mathrm{P}$ were calculated according to the assumption that the values $\mathrm{N}$ derive from [EMIM]P. [EMIM]P broke into the cellulose making strong hydrogen bond to cellulose and it was impossible to be extracted from the sample by rinsing with pure water. On the other hand, [EMIM]Br was completely extracted into the liquid phase, which is very attractive in the economic point of view because ionic liquid is quite expensive and should be recovered. However, about the decrease of crystallinity, [EMIM]P was far more effective than [EMIM]Br. This is probably because, although [EMIM]Br broke the structure of cellulose and solubilized once, cellulose was re-crystallized during recovery process. Contrary to this, [EMIM]P broke the structure and kept being captured, preventing re-crystallization of cellulose, which led to the better decrease of crystallinity. Considering the purpose of the pretreatment with ionic liquid was to convert the raw materials into more degradative precursors, [EMIM]P seems to satisfy this better. Furthermore, the TG curves confirmed this by showing the lower decomposition temperature for P-cedar.

\subsection{Hydrothermal Degradation of Pretreated Cedar}

The main products through the hydrothermal treatment were reducing sugar and organic acids. Figure 6 shows the carbon conversions into them on the basis of raw materials. The organic acids consist of formic and acetic acid for raw cedar, and succinic, glycolic and formic acid for pretreated cedar. The residue after the treatment was less for both pretreated cedar than raw sample cedar because of the loss of lignin during pretreatment, or because of the improved reactivity of samples through the pretreatment. Although there were some loss of lignin through the pretreatment, the yield of organic acids had increased, which indicates lignin surely had improved its reactivity according to the reports that lignin produces organic acids by hydrothermal treatment. However, about reducing sugar, Br-cedar produced less of it than raw sample. This was probably because cellulose in Br-cedar was stuck in lignin more rigidly than the raw material after the process of deposition. P-cedar increased the yield of reducing sugar as well as that of organic acids. The capture of [EMIM]P in its structure led to the decrease of crystallinity, and it seems to be one of the biggest factor for this increase, with the yield reaching $39 \mathrm{C}-\%$. It is now revealed that ionic liquid changes the structure of cedar, however, there is a problem of recovery. As mentioned in the previous section, there were some amounts of lignin extracted to ionic liquid. The separation of lignin from ionic liquid is very difficult, and it causes huge loss of

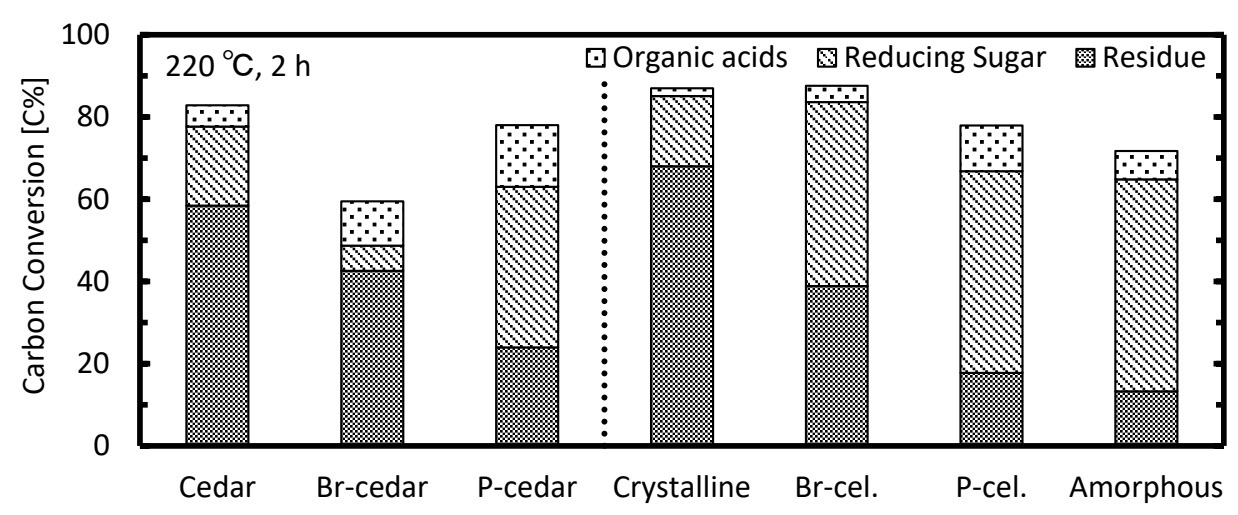

Fig. 6 Carbon conversions after hydrothermal treatment (on the basis of raw materials) 
ionic liquid. The high value of ionic liquid thus makes this process infeasible.

\subsection{Pretreatment of Crystalline Cellulose}

Because of the recovery issue of ionic liquid when cedar was used as the sample, conversion of cellulose was examined next. There are some established methods for the separation of cellulose from biomass (e.g., Li et al., 2009), so the refinery from biomass is still feasible. After the pretreatment of crystalline cellulose using [EMIM] Br at $80{ }^{\circ} \mathrm{C}$ for $6 \mathrm{~h}$, no solid cellulose was recognized in the sample before adding pure water. Then, by adding pure water, $99.0 \mathrm{wt} \%$ of cellulose was recovered as the deposit (Br-cellulose), without capturing [EMIM]Br. This fact that almost all cellulose was recovered after the pretreatment would be the confirmation for the hypothesis that [EMIM]Br did not extract any cellulose from cedar by the pretreatment (in the section 2.1). Figure 3, Figure 4 (b) and Figure 5 (b) show the compositions, the XRD patterns, and the TG curves of the samples, the products and also amorphous cellulose for comparison. Table 2 shows the elemental compositions of Br-cellulose. All the analyses showed quite similar tendencies to cedar's case. Crystallinity of crystalline cellulose decreased by about $30 \%$ through the pretreatment with [EMIM]Br judging from the XRD patterns. However, this decrease is not big considering that crystalline cellulose was completely solubilized once, so cellulose seemed to be recrystallized in the process of recovery. When the pretreatment with [EMIM] Br was conducted for $1 \mathrm{~h}$ or $3 \mathrm{~h}$ at $80^{\circ} \mathrm{C}$, the crystallinity decreased, respectively, by $0 \%$ and $22 \%$. Judging from the decreases between $1 \mathrm{~h}$ and $3 \mathrm{~h}$, and between $3 \mathrm{~h}$ and $6 \mathrm{~h}$, no decrease was expected for treatments longer than $6 \mathrm{~h}$. In addition, all the samples obtained by [EMIM]Br treatment at $120^{\circ} \mathrm{C}$ for $1 \mathrm{~h}, 3 \mathrm{~h}$ and $6 \mathrm{~h}$ showed similar XRD patterns and TG curves to those of obtained through $80{ }^{\circ} \mathrm{C}$ treatments, thus, the suitable condition for the [EMIM]Br pretreatment on crystalline cellulose was determined as $80{ }^{\circ} \mathrm{C}, 6 \mathrm{~h}$. Different from the pretreatment with [EMIM]Br, crystalline cellulose pretreated at $150{ }^{\circ} \mathrm{C}$ for $1 \mathrm{~h}$ with [EMIM]P was solubilized completely and then never recovered by adding pure water. Because when the pretreatment with [EMIM]P was conducted at $130{ }^{\circ} \mathrm{C}$, the mixed sample of crystalline cellulose and [EMIM]P was converted into gelatinous component and coagulated by adding pure water, the pretreatment temperature was determined as $150{ }^{\circ} \mathrm{C}$. Much ethanol was added to extract cellulose from the solution instead of pure water. The recovered deposit is donated as P-cellulose-deposit in this article, and the yield of the deposit was $130.0 \mathrm{wt} \%$. This means some amounts of [EMIM]P was recovered together with cellulose. It was clarified by CHNS elemental analysis and TG analysis that $72.4 \%$ of cellulose was recovered as P-cellulose-deposit and the rest of $57.6 \%$ derived from [EMIM]P(Figure 3 ). The XRD pattern and TG curve of P-cellulose-deposit are shown in Figure 4 (b) and Figure 5 (b), respectively. The elemental composition of P-cellulose-deposit is listed in Table 2. The crystallinity index of crystalline cellulose was very high comparing with cedar (about 5 times). Crystalline cellulose was uncrystallized very well and the thermal degradability increased by the pretreatment with [EMIM]P. Considering these results, P-cellulose-deposit seems to have improved its reactivity. However, the loss of cellulose during the pretreatment was not negligible. Besides, in the point of separation, solubilized cellulose was not necessarily separated from ionic liquid as the deposit before the following reactions because P-cellulose-deposit contained much ionic liquid to be separated later anyway. Thus, the following hydrothermal reaction was not conducted on P-cellulose-deposit but on P-cellulose, which is, the solubilized cellulose.

\subsection{Enzymatic Saccharification of Br-cellulose}

To confirm that crystalline cellulose was reformed to more degradative precursor, the enzymatic saccharification was conducted using crystalline cellulose, amorphous cellulose and Br-cellulose as samples. For the enzyme, Aspergillus was chosen. After the treatment at $50{ }^{\circ} \mathrm{C}$ for $24 \mathrm{~h}$, 8.2 C-\% of crystalline cellulose, 14.8 C-\% of amorphous cellulose and $15.6 \mathrm{C}-\%$ of Br-cellulose was converted into reducing sugar. As expected, cellulose increased its reactivity by the pretreatment. Crystallinity is assumed to be one of the biggest factors to effect on saccharification, however, Br-cellulose produced more glucose than amorphous cellulose. This means any other factor but cristallinity had bigger effect when Aspergillus was used as an enzyme. Although $24 \mathrm{~h}$ of saccharification time is usually not long enough for enzymatic saccharification, the purpose of this treatment was to confirm that crystalline cellulose became more degradative by the pretreatment, which was achieved.

2.5 Recovery of Br-cellulose with Subcritical Water

Br-cellulose was obtained by adding pure water to the solubilized crystalline cellulose with [EMIM]Br. However, the following process, saccharification, would be conducted by adding more water and the heating. In that case, the recovery of the solubilized crystalline cellulose with subcritical water possibly has the potential of direct saccharification. This recovery process was conducted using flow reaction system, by the solubilized cellulose being collided with the subcritical water. Because the viscosity of the 
solution was too high for the flow reaction system, DMSO was added to lower the viscosity of the solution before the treatment. At first, the effect of DMSO on the solution was examined by adding it at two different timings. One of them was adding DMSO to [EMIM]Br before the pretreatment and the other was adding DMSO to cellulose solution after the pretreatment. $98 \%$ of crystalline cellulose was solubilized when DMSO was added after the pretreatment while $22 \%$ was solubilized when DMSO was added before. This means DMSO had no effect on the deposition of solubilized cellulose but had the huge effect on the solubilization process. Because crystalline cellulose needed to be solubilized for the treatment with subcritical water, DMSO was added after the pretreatment as the preparation for the next process, and reducing sugar was obtained by 23.5 C-\% finally. Although this yield was higher than that obtained by enzymatic saccharification, it was not high enough to replace conventional process yet. In addition, DMSO was added in this process, which makes the separation more complicated. Thus, it was concluded that this direct degradation process was not preferable.

\subsection{Hydrothermal Degradation of Pretreated Crystalline Cellulose}

To confirm that [EMIM]P would not be spoiled by heat during the hydrothermal degradation, [EMIM]P was heated to higher temperature and confirmed that it starts degrading around $400{ }^{\circ} \mathrm{C}$. To examine the effect of crystallinity to the yield of reducing sugar, the hydrothermal degradation was conducted at $220{ }^{\circ} \mathrm{C}$ on crystalline and amorphous cellulose prior to the pretreated samples, and the result is shown in Figure 6. From the result, it is obvious that crystallinity had huge effect on the production of reducing sugar. Then, the hydrothermal degradation on the pretreated samples were conducted. Crystalline cellulose pretreated with both types of ionic liquid decreased its crystallinity and produced more reducing sugar than crystalline cellulose as shown in Figure 6. P-cellulose was converted into reducing sugar by almost the same yield as amorphous cellulose, reaching 50 C-\%. Br-cellulose produced more reducing sugar than crystalline cellulose, however, it was not uncrystallized enough to reach the yield from amorphous cellulose or P-cellulose. Next, the optimum reaction condition for hydrothermal treatment was sought. At first, the reaction temperature for the hydrothermal treatment was changed, and the result is shown in Figure 7 . Although Br-cellulose was converted into reducing sugar better than crystalline cellulose, the temperature degradation began at was almost the same. Furthermore, the yield was not as high as that from amorphous cellulose. However, crystalline cellulose pretreated with [EMIM]P increased the yield of reducing sugar significantly. The change in the degradation temperature was also remarkable, and the optimum hydrothermal temperature for saccharification was lowered to $150{ }^{\circ} \mathrm{C}$, reaching the yield of $90 \mathrm{C}-\%$. This yield was about the same as enzymatic saccharification reported by Dadi et al. (2006) through the treatment of $50 \mathrm{~h}$, and higher than the acidic saccharification reported by Li et al. (2006). The yield started decreasing as the temperature increased higher than $150{ }^{\circ} \mathrm{C}$. The products started producing residue at higher temperature than $150{ }^{\circ} \mathrm{C}$, which led to the decrease of the yield. Although it was obvious that crystallinity had huge effect on the production of reducing sugar, this high yield of [EMIM]P treatment cannot be explained only by crystallinity. Consequently, how [EMIM]P effects on crystalline cellulose or saccharides was investigated. At first, how glucose reacts to [EMIM]P through the treatment was examined. $0.1 \mathrm{~g}$ of glucose and $1.0 \mathrm{~g}$ of [EMIM]P were mixed in a glass bottle and put in an oil bath preheated to $150{ }^{\circ} \mathrm{C}$ for $1 \mathrm{~h}$ under stirring. Immediately after heating, the products were cooled, mixed with $8.9 \mathrm{~g}$ of pure water, and again heated at $150{ }^{\circ} \mathrm{C}$ for $2 \mathrm{~h}$. The product was analyzed by the DNS method and HPLC. According to the DNS analyses, the amount of reducing sugar detected was only $15.2 \%$ of initial amount. Different from that, the amount of reducing sugar detected by HPLC was even less, $4.3 \%$. For the confirmation, [EMIM]P was analyzed by the DNS method and it was revealed that [EMIM]P had no reduction ability. These facts indicate that equipped column for HPLC was not able to separate [EMIM]P and glucose in this pretreated sample, which did not happen to just the mixture of untreated [EMIM]P and glucose. In addition, it was also confirmed that there were no glucose degradants in the product such as 5-hydroxymethylfurfural or levulinic acid. In summary, what possibly happened was, glucose was polymerized or captured by

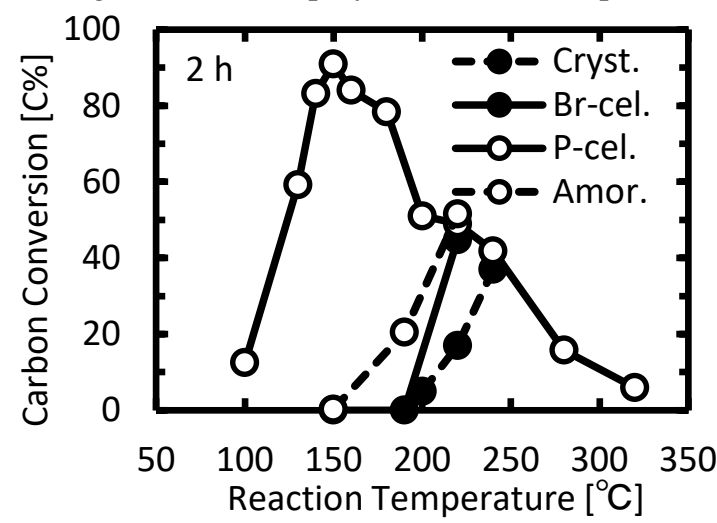

Fig. 7 Carbon conversion of P-cellulose into reducing sugar after hydrothermal treatment

(on the basis of raw materials) 
[EMIM]P too strongly to be separated through the reaction. However, considering the fact that reducing sugar was produced from P-cellulose by $90 \mathrm{C}-\%$ through the treatment, polymerization was unlikely to occur. When cellobiose and cellopentaose were analyzed by the DNS method, only cellobiose showed the output for each glucose unit while cellopentaose showed only $13 \%$. That means the product through the treatment on P-cellulose was mostly glucose or cellobiose, and the polymerization of them did not occur. Consequently, the valid explanation would be that glucose was captured by [EMIM]P. Then, there is a need of the explanation for the difference between P-cellulose and glucose that $90 \mathrm{C}-\%$ of P-cellulose was converted into reducing sugar while only $15.2 \mathrm{C}-\%$ of glucose was detected. For that, another experiment was conducted according to the same method using several types of samples consist of several different numbers of saccharide unit; glucose, cellobiose, fructo-oligosaccharide. The experiments without pretreatment were conducted according to the same method as well using $0.1 \mathrm{~g}$ of sample, $1.0 \mathrm{~g}$ of [EMIM]P and $8.9 \mathrm{~g}$ of pure water as samples. The result is shown in Figure 8. For the virgin samples, the result was reasonable with the yield increasing as molecular weight decreased. For the pretreated samples, on the other hand, the yield increased with molecular weight. Probably, this was because the samples were captured too strongly by [EMIM]P for the smaller molecular samples and they could not be extracted as reducing sugar anymore after the hydrothermal treatment. This two-step treatment method seems to be suitable for the bigger molecular samples such as cellulose. There is another need of the explanation for higher yield of reducing sugar from P-cellulose than virgin glucose. The first possible explanation is, some cellulose remained unsaccharified and captured by [EMIM]P, which lowered the capture ability of ionic liquid. However, this deactivation was unlikely to occur because

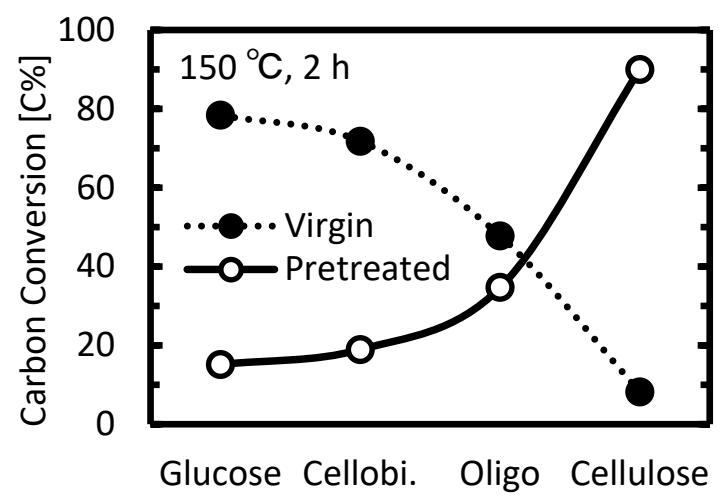

Fig. 8 Carbon conversion of different types of materials into reducing sugar after hydrothermal treatment excessive amount of [EMIM]P was used against crystalline cellulose in this reaction. The second possible explanation is, the capturing ability of [EMIM]P was lowered through the pretreatment. In this case, whether [EMIM]P still has the same performance when it was recycled would be a problem. Next, the hydrothermal degradation of P-cellulose was conducted for different reaction time at $150{ }^{\circ} \mathrm{C}$. The result is shown in Figure 9. The yield increased with reaction time. To obtain reducing sugar by more than $90 \mathrm{C}-\%$, the reaction time was required to be longer than 120 min. However, this reaction time is much shorter comparing with the enzymatic saccharification process.

\subsection{Recovery of Reducing Sugar and Ionic Liquid}

Ionic liquid was now revealed to be effective on the saccharification of cellulose, however, it is very expensive reagent and the recovery of ionic liquid is indispensable to make the process feasible. As shown in Figure 3, because P-cellulose-deposit trapped much [EMIM]P, it is better to recover ionic liquid all together after converting P-cellulose to reducing sugar by the hydrothermal degradation. For the recovery, the products of the hydrothermal treatment on P-cellulose was dried once to obtain the mixture of reducing sugar, unsaccharified cellulose and ionic liquid as residue, and then $20 \mathrm{~mL}$ of ethanol was added. This is because only ionic liquid dissolves into ethanol while the other components do not. The product was then filtered by suction, and the solution (which is supposed to contain ionic liquid and ethanol) was heated again to evaporate ethanol for the recovery of ionic liquid. The residue was mixed with pure water for the recovery of unsaccharified cellulose as residue and reducing sugar as solute by filtration, and then recovered components were dried in vacuo and analyzed by CHNS elemental analysis to determine the components. From the analyses, the water-soluble component was clarified to consist of mostly reducing sugar and a little amount of ionic liquid. The relationship between the recovery rate of

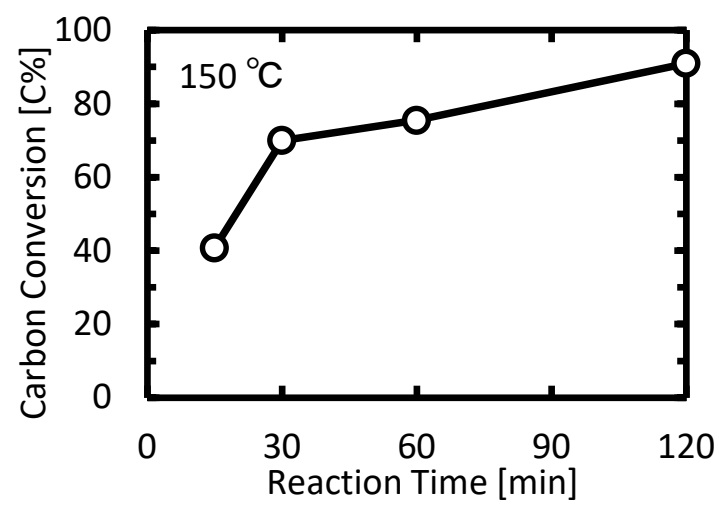

Fig. 9 Carbon conversion of P-cellulose into reducing sugar after hydrothermal treatment (on the basis of raw materials) 
reducing sugar or the loss of [EMIM]P as deposit and the reaction temperature is shown in Figure $\mathbf{1 0}$ with the carbon conversion into reducing sugar. The yields of ionic liquid and reducing sugar reached the highest at the reaction of $150{ }^{\circ} \mathrm{C}$, with the loss of ionic liquid reaching down to $1.7 \mathrm{wt} \%$. Considering the value of ionic liquid, $1.7 \%$ of loss is not small enough. However, the developed method would be only a part of whole process and further conversion of reducing sugar would help the recovery of ionic liquid, unless it inhibits the following conversions. Furthermore, the developed method which enabled the high conversion of cellulose into reducing sugar without using enzymes under mild conditions such as $2 \mathrm{~h}$ at $150{ }^{\circ} \mathrm{C}$ of the reaction might be a guide for the development of the cellulose conversion process.

\section{Conclusions}

An instructive conversion method of biomass into valuable chemical, reducing sugar, was developed for the utilization of biomass as an alternative resource to fossil resources. Ionic liquid was revealed to be an effective reagent on the production of reducing sugar from crystalline cellulose, decreasing its crystallinity. Comparing two types of ionic liquid, [EMIM]P was more effective than [EMIM]Br on the production from either samples used, crystalline cellulose and cedar. The conversion of cedar, however, was concluded as unpreferable because of the extraction of lignin by ionic liquid which requires a difficult separation. For the conversion of cellulose into reducing sugar, some experimental methods were examined for the enhancement of the yield, and significantly effective method was determined to be the hydrothermal degradation at $150{ }^{\circ} \mathrm{C}$ for $2 \mathrm{~h}$ after the pretreatment with [EMIM]P at $150{ }^{\circ} \mathrm{C}$ for $1 \mathrm{~h}$, with the yield reaching $90 \mathrm{C}-\%$. The recovery of the product and the reagent was also examined after the conversion of crystalline cellulose, and they were recovered, respectively, by $72.8 \%$ and $98.3 \%$. The recovery rate

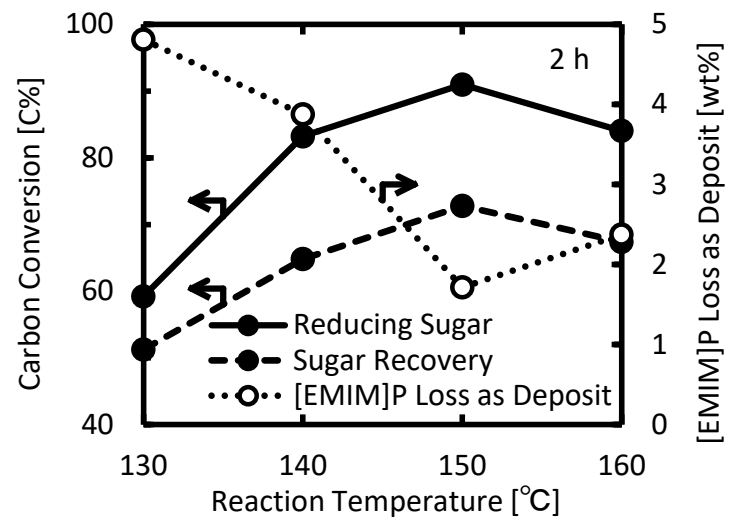

Fig. 10 Sugar recovery and loss of [EMIM]P after hydrothermal treatment (on the basis of raw materials) of ionic liquid ([EMIM]P) by $98.3 \%$ was not high enough because of its value, however, the higher recovery rate might be achieved if the process was followed by the further conversion of the product.

\section{Acknowledgements}

This work was financially supported by the Ministry of Education, Culture, Sports, Science and Technology of Japan through a Grant-in-Aid for Scientific Research (A) (Grant 25249109).

\section{Literature Cited}

Barthel, S. and T. Heinze; "Acylation and Carbanilation of Cellulose in Ionic Liquids," Green Chem., 8, 301-306 (2006)

Dadi, A. P., S. Varanasi and C. A. Schall; "Enhancement of Cellulose Saccharification Kinetics Using an Ionic Liquid Pretreatment Step,” Biotechnol. Bioeng., 95, 904-910 (2006)

Forsyth, S. A., J. M. Pringle and D. R. MacFarlane; "Ionic Liquids - An Overview," Aust. J. Chem., 57, 113-119 (2004)

Li. C. and Z. K. Zhao; "Efficient Acid-Catalyzed Hydrolysis of Cellulose in Ionic Liquid," Adv. Synth. Catal., 349, 1847-1850 (2007)

Li, R., J. Fei, Y. Cai, Y. Li, J. Feng and J. Yao; "Cellulose Whiskers Extracted from Mulberry: A Novel Biomass Production,” Carbohyd. Polym., 76, 94-99 (2009)

Liu, W., Y. Hou, W. Wu, S. Ren and W. Wang; "Complete Conversion of Cellulose to Water Soluble Substances by Pretreatment with Ionic Liquids,” Korean J. Chem. Eng., 29, 1403-1408 (2007)

Phillips, B. S. and J. S. Zabinski; "Ionic Liquid Lubrication Effects on Ceramics in a Water Environment,” Tribol. Lett., 17, 533-541 (2004)

Sievers, C., M. B. Valenzuela-Olarte, T. Marzialetti, I. Musin, P. K. Agrawal and C. W. Jones; "Ionic-Liquid-Phase Hydrolysis of Pine Wood," Ind. Eng. Chem. Res., 48, 1277-1286 (2009)

Swatloski, R. P., S. K. Spear, J. D. Holbrey and R. D. Rogers; "Dissolution of Cellulose with Ionic Liquids,” J. Am. Chem. Soc., 124, 4974-4975 (2002)

Uju, Y. Shoda, A. Nakamoto, M. Goto, W. Tokuhara, Y. Noritake, S. Katahira, N. Ishida, K. Nakashima, C. Ogino and N. Kamiya; "Short Time Ionic Liquids Pretreatment on Lignocellulosic Biomass to Enhance Enzymatic Saccharification,” Bioresource Technol., 103, 446-452 (2012)

Vo, H. T., Y. J. Kim, E. H. Jeon, C. S. Kim, H. S. Kim and H. Lee; "Ionic-Liquid-Derived, Water-Soluble Ionic Cellulose," Chem. Eur. J., 18, 9019-9023 (2012) 